\title{
Hypertriglyceridemia and Recurrent Pancreatitis following Splenectomy
}

\author{
Michael Butman ${ }^{a}$ David Taylor ${ }^{a} \quad K^{\prime}$ ristina Boström ${ }^{b}$ \\ Manuel Quinones ${ }^{c}$ Susanne B. Nicholas ${ }^{a, c}$ \\ aDivision of Nephrology, bivision of Cardiology, 'Division of Endocrinology, \\ Diabetes, and Hypertension, David Geffen School of Medicine at UCLA, \\ Los Angeles, Calif., USA
}

\section{Key Words}

Triglycerides · Pancreatitis · Splenectomy

\begin{abstract}
Hyperlipoproteinemia represents a constellation of clinical syndromes that frequently includes hypertriglyceridemia. Because of the degree of elevation in the triglyceride levels frequently seen in these syndromes, they are associated with complications not generally observed among those patients with essential hypertriglyceridemia, including as in this case report, recurrent pancreatitis. Here, we present a case of a patient with hyperlipoproteinemia who developed acute worsening of his hypertriglyceridemia and onset of acute panceatitis that became recurrent following elective splenectomy for suspected lymphoma. In particular, we discuss the dietary management of hypertriglyceridemia which significantly reduced the number of episodes of acute pancreatitis in this patient.
\end{abstract}

\section{Introduction}

The hyperlipoproteinemias are a collection of hereditary lipid disorders characterized by severely elevated serum lipid levels [1]. The particular pattern of lipid elevation (primarily triglycerides, or primarily cholesterol, or a combined picture) is determined by the particular inherited defect. For example, lipoprotein lipase (LPL) deficiency impairs the conversion of triglyceride-rich particles into cholesterol moieties such as intermediate-density lipoprotein (IDL) and low-density lipoprotein (LDL). LPL defects are thus primarily associated with hypertriglyceridemia, and in particular, chylomicronemia [1]. Other metabolic defects lead to other patterns of lipid elevation.

An uncommon, autosomal recessive deficiency in apolipoprotein CII results in impaired activity of LPL on chylomicrons and very low-density lipoprotein (VLDL).

Susanne B. Nicholas, MD, PhD FASN
900 Veteran Avenue, Suite 24-130, Los Angeles, CA 90095 (USA)

Tel. +1 310794 7555, Fax +1 3107947654

E-Mail sunicholas@mednet.ucla.edu 
Apolipoprotein CII is found on both chylomicrons and VLDL particles; this deficiency impairs the activity of LPL on these particles, preventing their conversion to IDL and LDL. As would be expected, this syndrome results in severe accumulation of the triglyceride-rich particles that can be very difficult to control even with aggressive medical treatment and lifestyle changes [1].

What is not well defined in the literature are adaptive responses the body undertakes in response to these extreme changes in the lipid profile. Although numerous consequences including accelerated atherosclerosis and foam cell formation, fatty liver, and splenomegaly have been described, they have generally been considered sequelae of the disease. Here, we present a case of hypertriglyceridemia with elevated chylomicrons and VLDL associated with massive splenomegaly that was acutely exacerbated by elective splenectomy.

\section{Case Report}

In January 1996, a 45-year-old white, male construction worker presented to his primary care physician for a routine annual physical examination. His medical and surgical history was significant for a cholecystectomy in 1982 due to cholecystitis that was believed to be secondary to clofibrate, which he was taking for hypercholesterolemia. He also had had an episode of acute pancreatitis in 1990 that was attributed to three weeks of heavy alcohol intake consisting of approximately six beers a day. He subsequently abstained from any further alcohol intake or tobacco use. The medical history was otherwise unremarkable. He took no other medications or herbal supplements. There was no family history of diabetes; his father was in good health and his mother had died of colon cancer. A review of systems was non-contributory, and the physical exam was unremarkable. Routine blood tests revealed an elevated blood glucose level consistent with diabetes, cholesterol of $271 \mathrm{mg} / \mathrm{dl}$, and a triglyceride level of $2,527 \mathrm{mg} / \mathrm{dl}$. He was diagnosed with adult onset diabetes and hyperlipidemia and was prescribed lifestyle modifications and gemfibrozil $600 \mathrm{mg}$ twice a day. In February 1996, after failing to control the elevated blood glucose with dietary changes, he was prescribed glipizide $5 \mathrm{mg}$ once a day and nizatidine $150 \mathrm{mg}$ twice a day. He subsequently began experiencing hypoglycemic episodes. Daily monitoring confirmed that blood glucose levels were consistently below $100 \mathrm{mg} / \mathrm{dl}$. As a result, the hypoglycemic agents were discontinued. Interestingly, once off hypoglycemic medications, he maintained stable fasting blood sugars that were subsequently paralleled with hemoglobin A1c values between 3.7 and 5.4. The cause of his history of hyperglycemia was never specifically identified.

On the other hand, control of the hyperlipidemia proved more elusive. Over the following six years, from January 1996 to May 2002, he was prescribed a variety of lipid-lowering agents but with limited degrees of success. In January 1996, he was first prescribed gemfibrozil; cholestyramine was added seven months later due to persistently elevated triglycerides. (The cholestyramine had to be replaced with colestipol after one month due to constipation.) In April 1998, an HMG-CoA reductase inhibitor was added but substituted with another after one month due to myalgias. This resulted in a reduction of total cholesterol to less than $200 \mathrm{mg} / \mathrm{dl}$, but the triglycerides remained elevated at 2,845 mg/dl. Nine months later gemfibrozil was discontinued due to leukopenia.

In June 1999, he was found to be hypothyroid and was started on thyroid replacement. Although the hypothyroidism was thought to contribute to his hyperlipidemia, both cholesterol and triglyceride progressively increased despite achieving a euthyroid state. By December 1999, cholesterol and triglyceride levels had increased to 344 and $4,044 \mathrm{mg} / \mathrm{dl}$, respectively. He was then started on fenofibrate, and niacin was later added.

From November 2000 until his hospitalization in May 2002 he remained on maximal doses of an HMG-CoA reductase inhibitor, fenofibrate, and niacin. During this period total cholesterol and triglyceride levels remained elevated and fluctuated between 335 and $450 \mathrm{mg} / \mathrm{dl}$, and between 4,390 and $5,380 \mathrm{mg} / \mathrm{dl}$, respectively.

In May 2002, he was hospitalized for abdominal pain with an enlarged spleen on exam. Abdominal ultrasound and CT scan confirmed massive splenomegaly, whereas liver size was normal; there was no comment regarding the presence or absence of a portal or splenic vein thrombosis. A complete evaluation, which included complete blood count with peripheral smear, bone marrow aspiration and biopsy, and rheumatologic serologies, revealed no obvious cause for the splenomegaly. Splenectomy was 
performed for persistent abdominal pain and suspicion of primary splenic lymphoma. However, the pathology showed only lipid-laden macrophages and no evidence of a malignant process. With improvement in his symptoms, he was discharged to outpatient follow-up for ongoing care including pneumococcal vaccination.

Four weeks after splenectomy, he presented to his physician's office complaining of left upper quadrant and severe upper abdominal pain with nausea. On physical examination, he was found to be normotensive and afebrile with a soft abdomen and some guarding but no rebound tenderness. The work-up included laboratories that revealed elevated amylase, lipase, and triglycerides; imaging to rule out a postoperative complications and thrombosis was not done at this time. This was the first of numerous episodes of pancreatitis due to hypertriglyceridemia that continued to plague him. Interestingly, following the splenectomy, both total cholesterol and triglyceride levels showed a dramatic rise; by April 2003, they had reached 1,020 and 11,290 mg/dl, respectively, at which time he was referred to UCLA (see table 1). Of note, the triglyceride-rich VLDL represented $98 \%$ of the total cholesterol on fractionation, that is, the patient had markedly elevated VLDL associated with low LDL and HDL. However, given the degree of elevation of the total triglyceride level, the severe hypertriglyceridemia measured was associated with, but not explained by, the elevated VLDL. This clinical picture suggested a hypertriglyceridemia syndrome characterized by elevated VLDL and severe chylomicronemia.

\section{Discussion}

Plasma triglycerides originate from either dietary sources or de novo hepatic synthesis. In the intestine, dietary triglycerides are packaged into chylomicrons. In the liver, triglyceride accumulation stimulates VLDL production. After a 12-hour fast, moderate hypertriglyceridemia (up to approximately $600 \mathrm{mg} / \mathrm{dl}$ ) may result from increased hepatic synthesis. When fasting triglyceride values exceed $600-800 \mathrm{mg} / \mathrm{dl}$ there is saturation of the triglyceride clearance process mediated by LPL. As a consequence of the saturation of the LPL system, chylomicron clearance is diminished, resulting in the accumulation of both chylomicrons and VLDL. This accumulation often leads to triglyceride levels of $1,000 \mathrm{mg} / \mathrm{dl}$ and higher. The presence of chylomicrons in association with triglyceride levels above $1,000 \mathrm{mg} / \mathrm{dl}$ is referred to as chylomicronemia syndrome [2]. If the LPL system is impaired, it may contribute to diminished clearance and chylomicronemia as well. This occurs in a variety of situations caused by acquired and/or genetic abnormalities of LPL or its lipoprotein receptor [1].

Interestingly, splenomegaly due to the accumulation of lipid-laden histiocytes is not associated with hypertriglyceridemia except in patients with chronic severe chylomicronemia. It is, however, found in other disorders in the notable absence of hypertriglyceridemia. These include cholesterol ester storage disease and lysosomal storage diseases such as Niemann-Pick variant, Gaucher disease, lecithin-cholesterol acyltransferase deficiency, and Tangier disease [3]. The clinical manifestations associated with these syndromes include skeletal, ophthalmologic, hematologic, neurologic, cardiac, and hepatic abnormalities. The case presented here did not have any of the aforementioned clinical abnormalities, suggesting that hypertriglyceridemia was most likely the cause of the splenomegaly.

Whether or not hypertriglyceridemia is independently associated with an increased risk of coronary artery disease is controversial [4]. For example, with rare exceptions, patients with chylomicronemia due to mutations in the LPL gene (type I lipoproteinemias) do not have an increased risk of cardiovascular disease despite total cholesterol and triglyceride levels as high as 1,000 and $10,000 \mathrm{mg} / \mathrm{dl}$, respectively [1]. On the other hand, patients with elevated levels of IDL and LDL or remnant dyslipidemia due to apoE mutations (types IIb and III lipoproteinemia, respectively) that are also characterized by high total cholesterol and triglyceride levels, have an increased risk of coronary artery disease $[5,6]$. The difference seems to be due to the lipoproteins involved. 
In chylomicronemia, the chylomicrons are thought to be so large that their entry into the subendothelial space is retarded so that arterial wall macrophages are not exposed to these large particles [7]. On the other hand, in patients with dyslipidemia due to elevated levels of LDL, IDL, and possibly VLDL, these smaller lipoproteins may have greater access to the subendothelial space where they can be ingested by macrophages that transform into atherogenic foam cells $[7,8]$. Given the low LDL and HDL, and the markedly elevated cholesterol and triglycerides (manifested as chylomicrons and VLDL), the patient presented here probably has type $\mathrm{V}$ lipoproteinemia most consistent with either LPL deficiency or a deficiency in apolipoprotein CII. Of note, types I and V have been associated with the same genetic defects and likely simply represent a variation in clinical presentation (see table 2).

Severe hypertriglyceridemia associated with chylomicronemia is a recognized risk factor for developing pancreatitis, but it only accounts for approximately $1.3-3.8 \%$ of all acute cases [9]. Typically, pancreatitis does not occur unless levels are greater than 1,000 $\mathrm{mg} / \mathrm{dl}[10]$. Although the mechanism is unknown, some have suggested that the liberation of free fatty acids by pancreatic lipase results in toxic injury to the capillary endothelium and pancreatic acinar cells $[10,11]$. In isolated perfused pancreatic preparations, for example, the addition of free fatty acids produced edema and hemorrhage in the pancreas [11]. There is some evidence to suggest that liberation of certain free fatty acids from triglyceride molecules may predispose to more severe episodes of pancreatitis. For example, increased levels of polyunsaturated free fatty acids and in particular, linoleic and arachidonic acids, were retrospectively associated with increased rates of complications from acute pancreatitis [12]. Since the choice of surgical or medical approach to the management of acute pancreatitis may depend on the presence or likelihood of developing complications [13], early evaluation of liberated free fatty acids, in patients with severe hypertriglyceridemia, may be of some benefit.

Even though hypertriglyceridemia is a risk factor for pancreatitis and dramatic triglyceride elevations have been reported after splenectomy, to our knowledge there is only one case report of pancreatitis due to hypertriglyceridemia following a splenectomy. In the letter to the editor, Schmidt et al. described the development of hypertriglyceridemia and a subsequent episode of pancreatitis in a patient following a traumatic splenectomy [14]. The triglyceride level was noted to rise markedly after the splenectomy and was recorded to be as high as $4,500 \mathrm{mg} / \mathrm{dl}$. As in the case reported by Schmidt et al., our case also demonstrates worsening hypertriglyceridemia and the development of pancreatitis following splenectomy. Furthermore, the presence of lipidladen macrophages in the splenic tissue following splenectomy seen on histopathology strongly suggested that the spleen served an important role as a site of chylomicron triglyceride clearance. Presumably, abolition of this clearance process resulted in a marked rise in serum triglycerides that led to recurrent episodes of pancreatitis.

Once the acute pancreatitis had resolved, control of the hypertriglyceridemia remained the goal of therapy. The typical management of hypertriglyceridemia consists of avoidance of alcohol, weight reduction, discontinuation of offending drugs, and control of diabetes mellitus and hypothyroidism [15]. It is important to note that fat restriction plays a pivotal role in management but must be performed in conjunction with exercise and weight reduction. Control of dietary fat intake, saturated and unsaturated, is the cornerstone of nutritional intervention. Dietary fat should be reduced to no more than 10 to $15 \%$ of total caloric consumption. If it does not adequately reduce triglycerides and prevent pancreatitis, then consideration should be given to using medium chain triglycerides, which bypass chylomicron formation and are taken up from the portal vein 
by the liver. In addition, supplementation with fish oil can decrease endogenous production of triglyceride-rich lipoproteins. It has been shown that daily intake of 3-4 g of fish oil, consisting of equal amounts of eicosapentaenoic and docosahexaenoic acids, will decrease plasma triglyceride levels by $30-50 \%$ in patients with hypertriglyderidemia $[16,17]$. Drug treatment with a fibric acid derivative (gemfibrozil, fenofibrate or clofibrate) or niacin may help to decrease triglyceride levels by limiting hepatic production of VLDL. However, niacin use may be limited by its known side effects (flushing, pruritus, hepatotoxicity, glucose intolerance, hyperuricemia and gastric upset). HMG-CoA reductase inhibitors are not primarily used in the management of hypertriglyceridemia.

In this patient, treatment consisted of adhering to a strict diet limited to less than $20 \mathrm{~g}$ of fat per day in combination with daily doses of fenofibrate $(160 \mathrm{mg})$, atorvastatin (40 $\mathrm{mg})$, fish oil (6 g), and niacin (1,000 mg). Following this strict dietary and medical regimen, the patient's cholesterol and triglyceride were reduced to 630 and 3,940 mg/dl, respectively and recurrent episodes of acute pancreatits resolved.

\section{Summary}

We believe that our patient developed worsening hypercholesterolemia and hypertriglyceridemia as a result of his splenectomy. It appears that the spleen served as a site of storage for lipids. This is supported by the pathological changes in the spleen and the concomitant rise in serum lipids following its removal. As a result, this sudden rise in serum triglycerides was the cause of the recurrent episodes of pancreatitis.

The case presented here illustrates the challenges encountered by physicians when managing and treating patients with lipid disorders. Physicians need to be familiar with the different types of lipid abnormalities, the clinical manifestations and laboratory findings, and the different treatments available, whether there are dietary modifications, the use of pharmaceutical agents, or a combination of the two. Furthermore, it is necessary that physicians be aware of the complications associated with lipid disorders, including those associated with their treatment, such as side effects of the medication, or, as in the case here, the development of pancreatitis following splenectomy.

\section{Acknowledgement}

Drs. Michael Butman and David Taylor were supported by NIH training grant T32-DK07789. 
Table 1. Cholesterol and triglyceride levels from $11 / 2000$ through $3 / 2004$. Splenectomy was performed in May $2002\left(^{*}\right)$ after which lipids proceeded to rise to the highest levels recorded

\begin{tabular}{|c|c|c|c|c|c|c|c|c|c|c|c|c|}
\hline & $11 / 00$ & $5 / 01$ & $12 / 01$ & $5 / 02^{*}$ & $6 / 02$ & $7 / 02$ & $8 / 02$ & $11 / 02$ & $1 / 03$ & $2 / 03$ & $4 / 03$ & $3 / 04$ \\
\hline Cholesterol, mg/dl & 440 & 335 & 350 & 218 & 211 & 970 & 850 & 530 & 700 & 840 & 1,020 & 630 \\
\hline Triglyceride, $\mathrm{mg} / \mathrm{dl}$ & 4,760 & 4,800 & 5,380 & 1,300 & 626 & 6,940 & 10,410 & 3,030 & 8,780 & 8,190 & 11,290 & 3,940 \\
\hline
\end{tabular}

Table 2. Phenotypes and classification of hyperlipidemic syndromes

\begin{tabular}{lllll}
\hline Elevated moiety & $\begin{array}{l}\text { Hyperlipidemia } \\
\text { classification }\end{array}$ & Common pathogenic defects & Disease name & Findings and symptoms \\
\hline Chylomicrons & I & $\begin{array}{l}\text { Lipoprotein lipase deficiency } \\
\text { Apolipoprotein CII deficiency }\end{array}$ & $\begin{array}{l}\text { Familial LPL deficiency } \\
\text { Familial CII deficiency }\end{array}$ & $\begin{array}{l}\text { Pancreatitis, eruptive } \\
\text { xanthomas (trunk and } \\
\text { extremities), and } \\
\text { hepatosplenomegaly }\end{array}$ \\
\hline $\begin{array}{l}\text { Chylomicrons } \\
\text { and VLDL }\end{array}$ & V & $\begin{array}{l}\text { Lipoprotein lipase deficiency } \\
\text { Apolipoprotein CII deficiency }\end{array}$ & $\begin{array}{l}\text { Familial LPL deficiency } \\
\text { Familial CII deficiency }\end{array}$ & $\begin{array}{l}\text { Pancreatitis, eruptive } \\
\text { xanthomas (trunk and } \\
\text { extremities), and } \\
\text { hepatosplenomegaly }\end{array}$ \\
\hline VLDL & IV & Unknown & Familial hypertriglyceridemia & $\begin{array}{l}\text { As above; may have increased } \\
\text { vascular risk }\end{array}$ \\
\hline VLDL), IDL & III & Apolipoprotein E mutation & Familial dysbetalipoproteinemia & $\begin{array}{l}\text { Vascular disease, tuberous } \\
\text { xanthomas }\end{array}$ \\
\hline VLDL, LDL & IIB & Multiple & Familial combined hyperlipidemia & Vascular disease \\
\hline LDL & IIA & Multiple & Familial combined hyperlipidemia & $\begin{array}{l}\text { Vascular disease } \\
\text { Dascular disease, tuberous and } \\
\text { tendinous xanthomas }\end{array}$ \\
\hline
\end{tabular}

*E2 is the defective allele of apolipoprotein $\mathrm{E}$, which cannot bind its receptor.

Adapted from [1]. 


\section{References}

1 Ginsberg HN, Goldberg IJ: Disorders of lipoprotein metabolism; in Fauci A et al. (eds): Harrison’s Principles of Internal Medicine. New York, McGraw Hill, 1998, pp 2138-2149.

2 Chait A, Brunzell JD: Chylomicronemia syndrome. Adv Intern Med 1992;37:249273.

3 Nguyen TT, et al: Familial splenomegaly: macrophage hypercatabolism of lipoproteins associated with apolipoprotein $\mathrm{E}$ mutation [apolipoprotein $\mathrm{E}$ (delta149 Leu)]. J Clin Endocrinol Metab 2000;85:4354-4358.

4 Criqui $\mathrm{MH}$, et al: Plasma triglyceride level and mortality from coronary heart disease. N Engl J Med 1993;328:1220-1225.

5 Assmann G, Brewer HB Jr: Genetic (primary) forms of hypertriglyceridemia. Am J Cardiol 1991;68:13A-16A.

6 Fung MA, Frohlich JJ: Common problems in the management of hypertriglyceridemia. CMAJ 2002;167:1261-1266.

7 Benlian P, et al: Premature atherosclerosis in patients with familial chylomicronemia caused by mutations in the lipoprotein lipase gene. N Engl J Med 1996;335:848-854.

8 Li AC, Glass CK: The macrophage foam cell as a target for therapeutic intervention. Nat Med 2002;8:1235-1242.

9 Athyros VG, et al: Long-term follow-up of patients with acute hypertriglyceridemia-induced pancreatitis. J Clin Gastroenterol 2002;34:472-475.

10 Toskes PP: Hyperlipidemic pancreatitis. Gastroenterol Clin North Am 1990;19:783-791.

11 Saharia P, et al: Acute pancreatitis with hyperlipemia: studies with an isolated perfused canine pancreas. Surgery 1977;82:60-67.

12 Sztefko K, Panek J: Serum free fatty acid concentration in patients with acute pancreatitis. Pancreatology 2001;1:230-236.

13 Andersson B, et al: Severe acute pancreatitis - outcome following a primarily non-surgical regime. Pancreatology 2006;6:536-541.

14 Schmidt HH, Wagner S, Manns M: The spleen as a storage pool in lipid metabolism. Am J Gastroenterol 1997;92:1072.

15 Yadav D, Pitchumoni CS: Issues in hyperlipidemic pancreatitis. J Clin Gastroenterol 2003;36:54-62.

16 Harris WS: Fish oils and plasma lipid and lipoprotein metabolism in humans: a critical review. J Lipid Res 1989;30:785-807.

17 Pschierer V, Richter WO, Schwandt P: Primary chylomicronemia in patients with severe familial hypertriglyceridemia responds to long-term treatment with (n-3) fatty acids. J Nutr 1995;125:1490-1494. 